\title{
EFEITO DE EMBALAGENS COM ATMOSFERA MODIFICADA SOBRE OS ASPECTOS MICROBIOLÓGICOS E SENSORIAIS DE GOIABAS MINIMAMENTE PROCESSADAS
}

\author{
PALHARINI, Maria Cecília de Arruda ${ }^{1}$ \\ JERÔNIMO, Elisangela Marques ${ }^{2}$ \\ JACOMINO, Angelo Pedro ${ }^{3}$ \\ FILETI, Mirian de Souza ${ }^{4}$ \\ ALMEIDA, Aparecida Marques de ${ }^{1}$
}

\begin{abstract}
RESUMO: O objetivo deste trabalho foi avaliar o efeito de diferentes embalagens com atmosfera modificada na qualidade microbiológica e sensorial de goiabas 'Kumagai' e 'Pedro Sato' minimamente processadas. Mix de goiabas 'Kumagai' e 'Pedro Sato', em rodelas, foram embaladas em diferentes materiais de embalagem e armazenadas a $5^{\circ} \mathrm{C}, 70 \% \mathrm{UR}$ por seis dias. Os materiais de embalagem utilizados foram: embalagens de tereftalato de polietileno (PET); filme de polipropileno de $52 \mu \mathrm{m}$ de espessura (PP); filme poliolefínico da Cryovac de 58 $\mu \mathrm{m}$ (PD900); filme de policloreto de vinil de $17 \mu \mathrm{m}$ (PVC). Avaliou-se a contagem de microrganismos acidúricos, coliformes totais e à $45^{\circ} \mathrm{C}$ e presença/ausência de Salmonella. Para avaliação sensorial aplicou-se o teste de aceitação. As goiabas minimamente processadas e acondicionadas em embalagem de PVC e PP obtiveram maior aceitação sensorial referente ao atributo aparência. A aceitação sensorial referente ao atributo sabor das goiabas minimamente processadas foi positiva em todos os tratamentos. A embalagem não influenciou os aspectos microbiológicos dos produtos, que apresentaram baixa contagem microbiana, em acordo com a legislação vigente.
\end{abstract}

Palavras-chave: Psidium guajava. Processamento mínimo. Microbiologia. Aparência. Sabor.

\section{EFFECT OF PACKAGING WITH MODIFIED ATMOSPHERE ON MICROBIOLOGICAL AND SENSORIAL ASPECTS OF MINIMALLY PROCESSED GUAVAS}

SUMMARY: The aim of this study was to evaluate the effect of different packaging with modified atmosphere on the microbiological aspects and sensory acceptance of minimally processed 'Kumagai' and 'Pedro Sato' guavas. Mix of 'Kumagai' and 'Pedro Sato, in slices were wrapped in different packaging materials and stored at $5^{\circ} \mathrm{C}$ for six days. The packaging materials used were:polyethylene terephthalate containers; polypropylene bag $52 \mu \mathrm{m}$; polyolefin film Cryovac PD-900 $58 \mu \mathrm{m}$; PVC stretch film $17 \mu \mathrm{m}$. Aciduric microorganisms, total coliforms and at $45^{\circ} \mathrm{C}$ and the presence / absence of Salmonella were analyzed. For the sensory evaluation was applied to the acceptance test. The guavas minimally processed wrapped PVC stretch film and PP film obtained greater acceptance for the sensory attribute appearance. The acceptance for the sensory attribute flavor of minimally processed guavas was positive in all treatments. The packaging did not affect the microbiological aspects of products, which showed low microbial count, in accordance with current legislation.

Keywords: Psidium guajava. Minimal processing. Microbiology. Appearance. Flavor.

\section{INTRODUÇÃO}

$\mathrm{O}$ mercado de frutas minimamente processadas tem grande potencial de crescimento, visto a necessidade das pessoas em adquirirem produtos frescos e convenientes. As pessoas são motivadas a consumirem frutas, pois estas fazem bem à saúde, têm valor nutricional elevado e sabor agradável (JACOMINO; ARRUDA; MOREIRA, 2005). A goiaba vermelha destaca-se por ser rica em licopeno enquanto a goiaba branca destaca-se por ser rica em vitamina $\mathrm{C}$, com teores superiores ao da laranja. De

\footnotetext{
1 Pesquisador Científico, APTA- Pólo Regional Centro Oeste, Av. Rodrigues Alves, 40-40, 17030-000, Bauru/SP; mcarruda@apta.sp.gov.br; ${ }^{2}$ Pesquisador Científico, APTA- Pólo Centro Oeste, Unidade de Pesquisa de Jaú, Rodovia Deputado Leônidas Pacheco Ferreira, Km 304, Cx.P. 66, 17201-970, Jaú/SP; ${ }^{3}$ Prof. Dr. do Departamento de Produção Vegetal da ESALQ/USP, Av. Pádua Dias, 11, 13418-900 - Piracicaba/SP ${ }^{4}$ Graduanda do curso de Agronomia da FAEF, Garça/SP.
} 
acordo com a Tabela de Composição dos Alimentos (NEPA, 2006) o teor de vitamina C da goiaba branca é 99,2 mg $100 \mathrm{~g}^{-1}$ contra 53,7 da laranja.

O Brasil é um dos principais países produtores de goiaba cuja produção anual é cerca de 350 mil toneladas (FNP, 2010). Deste montante, a maior parte é destinada à industrialização. Diante disso, o processamento mínimo de goiabas, buscando associar variedades de polpa branca com as de polpa vermelha é uma forma de tornar a fruta mais atrativa e competitiva, atingindo um nicho diferenciado do mercado consumidor (JACOMINO et al., 2007).

O processamento promove danos físicos nos tecidos vegetais, tornando os produtos minimamente processados mais perecíveis que os produtos intactos (ARTES; GÓMEZ; HERNÁNDEZ, 2007) e mais suscetíveis à infecção por microrganismos (BRECHT, 1995).

A vida útil de produtos minimamente processados pode ser prolongada desde que técnicas adequadas de conservação compatíveis com o produto a ser armazenado sejam adotadas. A refrigeração aliada à atmosfera modificada é amplamente utilizada na conservação de produtos minimamente processados (FARBER, 1991).

A utilização de embalagens com atmosfera modificada torna-se interessante, por apresentar efeitos diretos nos processos fisiológicos e bioquímicos do vegetal minimamente processado, bem como na redução da proliferação microbiana e desse modo aumentar a vida útil dos vegetais.

Este trabalho teve como objetivo verificar o efeito de diferentes embalagens com atmosfera modificada na qualidade microbiológica e sensorial de goiabas 'Kumagai' e 'Pedro Sato'minimamente processadas.

\section{MATERIAL E MÉTODOS}

As goiabas brancas 'Kumagai' foram provenientes de pomar comercial com idade de 20 anos, situado em Campinas/SP (22 $54^{\prime} \mathrm{S} ; 4^{\circ}$ 03' O) e as goiabas vermelhas 'Pedro Sato' foram provenientes de pomar comercial com idade de cinco anos, situado em Itajú/SP ( $\left.21^{\circ} 58^{\prime} \mathrm{S} ; 48^{\circ} 48^{\prime} \mathrm{O}\right)$. O sistema de condução de ambos os pomares era em taça aberta, com 4 a 6 pernadas.

As frutas foram selecionadas quanto à ausência de danos mecânicos e grau de maturação (coloração do epicarpo verde clara, início da coloração amarela correspondente ao estádio 2 de maturação). As frutas foram lavadas em água corrente e imersas em solução clorada $\left(200 \mathrm{mg} \mathrm{L}^{-1}\right)$ por 10 minutos. Posteriormente permaneceram durante 12 horas em câmara fria a $10^{\circ} \mathrm{C}$ para então serem submetidas ao processamento, seguindo-se os procedimentos adequados de higiene (Boas Práticas de Fabricação).

Porções de aproximadamente $220 \mathrm{~g}$ de produto minimamente processado em rodelas, de goiabas 'Kumagai' e 'Pedro Sato' foram acondicionadas em bandejas de poliestireno expandido ou embalagem rígida, constituindo um mix de goiaba.

As bandejas foram recobertas com diferentes materiais de embalagem, constituindo diferentes tratamentos: filme de policloreto de vinil (PVC) de $17 \mu \mathrm{m}$ de espessura; filme de polipropileno (PP) de 52 $\mu \mathrm{m}$ de espessura; filme poliolefínico da Cryovac (PD-900) de $58 \mu \mathrm{m}$ de espessura.

A embalagem rígida utilizada foi de tereftalato de polietileno (PET) com tampa do mesmo material, sem o revestimento com filmes.

As bandejas de poliestireno expandido (Isopor) (Cryovac, modelo 2F) utilizadas foram de $21 \mathrm{~cm}$ de comprimento, $14 \mathrm{~cm}$ de largura e $2 \mathrm{~cm}$ de altura e as embalagens de tereftalato polietileno (PET) (Plaszom, modelo 06), com capacidade de $500 \mathrm{ml}, 12 \mathrm{~cm}$ de diâmetro e 7,5 cm de altura.

Os produtos foram armazenados à $5^{\circ} \mathrm{C}, 70 \%$ UR durante um período de seis dias, sendo avaliados a cada três dias quanto aos aspectos microbiológicos e sensoriais.

Nucleus, v.9, n.1, abr.2012 
As análises microbiológicas foram realizadas conforme Silva et al. (2007). Em relação aos microrganismos acidúricos (bolores e leveduras e bactérias acidúricas) por plaqueamento em BDA, incubação a $25^{\circ} \mathrm{C}$ por 3-5 dias. Para pesquisa de coliformes totais e à $45^{\circ} \mathrm{C}$ foi utilizada a técnica do Número Mais Provável. A detecção de Salmonella foi realizada através de método oficial aprovado pela AOAC (Association of Official Analytical Chemists) para utilização em todos os tipos de alimentos, o qual utiliza o kit '1-2 test' fabricado pela Bio-control/USA.

A avaliação sensorial foi realizada aplicando-se o teste de aceitação sobre os atributos aparência e sabor, por 30 julgadores não treinados, utilizando-se escala estruturada de 9 pontos (9-gostei extremamente; 1-desgostei extremamente) Avaliou-se a intenção de compra do produto, mediante escala estruturada de 5 pontos (1- certamente compraria; 5- certamente não compraria), conforme Stone \& Sidel (1993).

Para avaliação de sabor as goiabas minimamente processadas foram apresentadas em porções de $20 \mathrm{~g}$ em copos descartáveis, codificados com números de 3 dígitos. Para análise de aparência e intenção de compra, as goiabas minimamente processadas foram apresentadas nas embalagens originais. As amostras foram servidas em laboratório com cabines individuais, de forma monádica.

$\mathrm{O}$ delineamento foi o inteiramente casualizado, em esquema fatorial (embalagens $\mathrm{x}$ tempo de armazenamento). Os resultados da análise microbiológica foram comparados com a legislação vigente (BRASIL, 2001). Os resultados da análise sensorial foram submetidos à análise de variância e teste de comparação de médias (Tukey 5\%).

\section{RESULTADOS E DISCUSSÃO}

Não foram detectados coliformes totais e a $45^{\circ} \mathrm{C}$ e nem Salmonella, estando portanto, as amostras de todos os tratamentos de acordo com a RDC n ${ }^{\circ} 12$ (BRASIL, 2001).

De acordo com a International Commission on Microbiological Specifications for Foods - ICMSF (1978), a presença de coliformes em alimentos indica manipulação inadequada durante o processamento, uso de equipamentos em más condições sanitárias ou ainda utilização de matéria-prima contaminada. Assim como os coliformes, a Salmonella pertence à família das Enterobacteriaciae e os principais focos de infecção são as fezes humanas e de animais. Portanto, a ausência destes microrganismos nas goiabas minimamente processadas é reflexo da ótima qualidade da matéria-prima utilizada e das boas práticas de fabricação adotadas durante todo o processamento.

Farber (1991) relata que a alteração da composição gasosa ao redor do produto pelo uso de embalagens influencia o desenvolvimento de microrganismos. Neste trabalho a atmosfera modificada não alterou a microbiota das goiabas minimamente processadas. A contagem de microrganismos acidúricos foi baixa, com valores na ordem de $10^{1}$ est $\mathrm{UFC} \mathrm{g}^{-1}$ (est $=$ valores estimados, placas com menos de 25 colônias), em todos os tratamentos.

Mattiuz, Durigan e Rossi Júnior (2003) também verificaram boa qualidade microbiológica de goiabas minimamente processadas durante sete dias a $3^{\circ} \mathrm{C}$ e atribuem esse fato à acidez elevada da fruta, à temperatura de refrigeração e às boas práticas de fabricação adotadas durante o processamento.

O mix de goiabas embalado em filme PVC apresentou maior valor referente às notas da avaliação sensorial para o atributo aparência até o terceiro dia de armazenamento. Entretanto, não diferiu estatisticamente em relação às notas atribuídas para as goiabas embaladas em filme PP (Tabela 1). A maior aceitação pelo filme PVC, provavelmente ocorreu em consequência deste material não favorecer a condensação do vapor d'água em sua superfície. Em relação ao filme PP, o brilho e transparência do material contribuíram para maior aceitação pelos provadores.

No sexto dia de armazenamento a aparência das goiabas minimamente processadas acondicionada 
nas diferentes embalagens não diferiu entre si. Nestas condições observou-se que as goiabas apresentaram sinais de ressecamento mais aparente nas embalagens PVC e PP.

Tabela 1. Média* das notas de aceitação** para o atributo aparência de goiabas 'Kumagai' e 'Pedro Sato' minimamente processadas em rodelas, acondicionadas em diferentes embalagens e armazenadas a $5^{\circ} \mathrm{C}$.

\begin{tabular}{cccc}
\hline Embalagens/Filmes & \multicolumn{3}{c}{ Dias de armazenamento a $5^{\circ} \mathrm{C}$} \\
\cline { 2 - 4 } & 0 & 3 & 6 \\
\hline PVC & $7,37 \mathrm{a}$ & $7,40^{\mathrm{a}}$ & $6,13 \mathrm{a}$ \\
PD-900 & $5,67 \mathrm{~b}$ & $5,57 \mathrm{~b}$ & $5,93 \mathrm{a}$ \\
PP & $6,40 \mathrm{ab}$ & $6,27 \mathrm{ab}$ & $5,37 \mathrm{a}$ \\
PET & $5,33 \mathrm{~b}$ & $5,43 \mathrm{~b}$ & $5,93 \mathrm{a}$ \\
\hline
\end{tabular}

* Médias seguidas de mesma letra na coluna não diferem entre si ao nível de 5\% de probabilidade; **Notas: 9=gostei extremamente; 8=gostei muito; 7=gostei moderadamente; $6=$ gostei ligeiramente; $5=$ nem gostei/nem desgostei; $4=$ desgostei ligeiramente; $3=$ desgostei moderadamente; $2=$ desgostei muito; $1=$ desgostei extremamente.

Em relação ao atributo sabor, as goiabas acondicionadas nos diferentes tipos de embalagem foram aceitas sensorialmente até o sexto dia de armazenamento a $5^{\circ} \mathrm{C}$. As notas variaram entre 8 ("gostei muito"), no dia do processamento a 6 ("gostei ligeiramente"), no sexto dia de armazenamento (Tabela 2).

Tabela 2. Média* das notas de aceitação** para o atributo sabor de goiabas 'Kumagai' e 'Pedro Sato' minimamente processadas em rodelas, acondicionadas em diferentes embalagens e armazenadas a $5^{\circ} \mathrm{C}$.

\begin{tabular}{cccc}
\hline Embalagens/Filmes & \multicolumn{3}{c}{ Dias de armazenamento a $5^{\circ} \mathrm{C}$} \\
\cline { 2 - 4 } & 0 & 3 & 6 \\
\hline PVC (branca) & $7,80 \mathrm{a}$ & $6,57^{\mathrm{a}}$ & $6,47 \mathrm{a}$ \\
PVC (verm) & $7,53 \mathrm{a}$ & $6,33^{\mathrm{a}}$ & $5,93 \mathrm{a}$ \\
PD-900 (branca) & $7,80 \mathrm{a}$ & $6,37^{\mathrm{a}}$ & $6,4 \mathrm{a}$ \\
PD-900 (verm) & $7,53 \mathrm{a}$ & $5,80 \mathrm{ab}$ & $6,07 \mathrm{a}$ \\
PP (branca) & $7,80 \mathrm{a}$ & $6,57^{\mathrm{a}}$ & $6,63 \mathrm{a}$ \\
PP (verm) & $7,53^{\mathrm{a}}$ & $6,33^{\mathrm{a}}$ & $6,30 \mathrm{a}$ \\
PET (branca) & $7,80^{\mathrm{a}}$ & $6,57^{\mathrm{a}}$ & $6,07 \mathrm{a}$ \\
PET (verm) & $7,53^{\mathrm{a}}$ & $5,10 \mathrm{~b}$ & $6,37 \mathrm{a}$ \\
\hline
\end{tabular}

* Médias seguidas de mesma letra na coluna não diferem entre si ao nível de 5\% de probabilidade; **Notas: 9=gostei extremamente; $8=$ gostei muito; $7=$ gostei moderadamente; $6=$ gostei ligeiramente; $5=$ nem gostei/nem desgostei; $4=$ desgostei ligeiramente; $3=$ desgostei moderadamente; $2=$ desgostei muito; $1=$ desgostei extremamente.

Quanto à intenção de compra, o mix de goiabas embaladas em PVC foi o mais aceito, com maior frequência das notas entre 'certamente compraria' e 'provavelmente compraria' (Figura 1).

Figura 1 - Teste de intenção de compra das goiabas minimamente processadas em rodelas e acondicionadas em diferentes embalagens (1-certamente compraria; 2-provavelmente compraria; 3-talvez compraria; 4-provavelmente não compraria; 5- certamente não compraria).

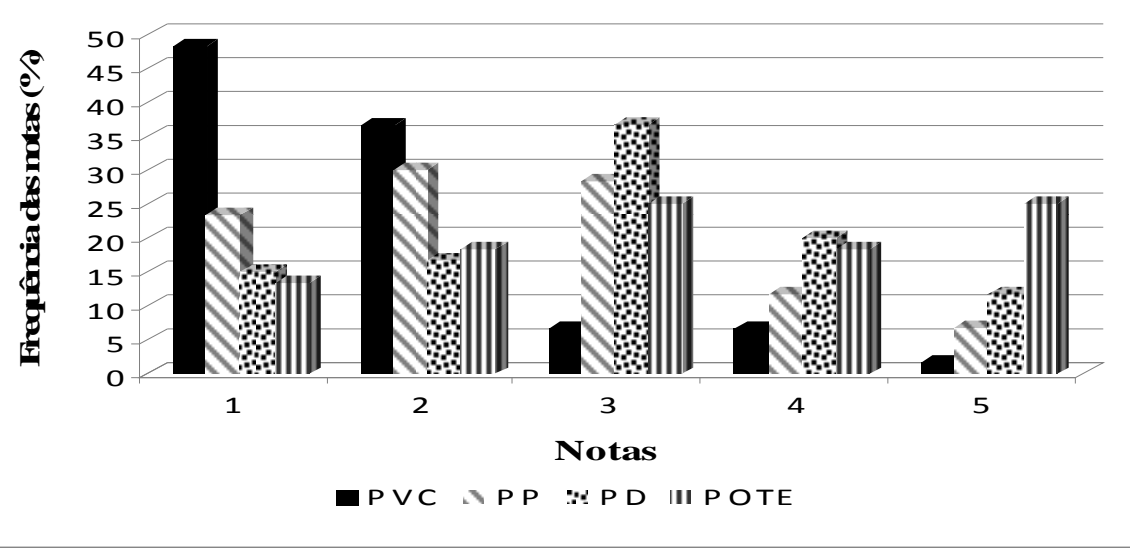




\section{CONCLUSÃO}

As goiabas minimamente processadas e embaladas em filme PVC e PP obtiveram maior aceitação sensorial referente ao atributo aparência.

A aceitação sensorial referente ao atributo sabor das goiabas minimamente processadas foi positiva em todos os tratamentos.

Não houve efeito do tipo de embalagem na proliferação microbiana.

\section{AGRADECIMENTOS}

Ao Conselho Nacional de Desenvolvimento Científico e Tecnológico (CNPq) pelo apoio financeiro ao projeto.

\section{REFERÊNCIAS}

ARTES, F.; GÓMEZ, P.A.; HERNÁNDEZ, F.A. Physical, physiological and microbial deterioration of minimally fresh processed fruits and vegetables. Food Science Technology International, v.13, n.3, p.177-188, 2007.

BRASIL. Resolução RDC n. 12 de 02 de janeiro de 2001 da Agência Nacional de Vigilância Sanitária. Dispõe sobre o Regulamento técnico sobre padrões microbiológicos para alimentos. Diário Oficial da República Federativa do Brasil, Brasília, DF, 10 jan. 2001.

BRECHT, J.K. Physiology of lightly processed fruits and vegetables. HortScience, v.30, p.18-21, 1995.

FARBER, J.M. Microbiological aspects of modified atmosphere packaging technology - A review.

Journal of Food Protection, v.54, n.1, p.58-70, 1991.

FNP CONSULTORIA \& COMÉRCIO. Agrianual 2010: anuário estatístico da agricultura brasileira. São Paulo, 341-344: Goiaba. 2010.

INTERNATIONAL COMMISSION ON MICROBIOLOGICAL SPECIFICATIONS FOR FOODS. Microorganisms in foods. 2. ed. Toronto: University of Toronto Press, 1978. v.1, 434p.

JACOMINO, A. P.; ARRUDA, M. C.de; MOREIRA, R. C.Tecnologia de processamento mínimo de frutas cítricas In: CONFERÊNCIA INTERNACIONAL DE CIENCIA Y TECNOLOGIA DE ALIMENTOS, 2005, La Habana. Nuevas tecnologías de conservación y envasado de frutas y hortalizas...p.11-17.

JACOMINO, A. P.et al. Goiaba e frutas cítricas: opções para o processamento mínimo. In: LOBO, M.G.; GONZÁLES, M. (Ed.). Processamento Mínimo de Frutas. Cartagena: Sabater, 2007. p. 55 - 69.

MATTIUZ, B.; DURIGAN, J. F.; ROSSI JUNIOR, O. D. Processamento mínimo em goiabas 'Paluma' e 'Pedro Sato'. 2. Avaliação química, sensorial e microbiológica. Ciência e Tecnologia de Alimentos, v.23, n.3, p.409-413, 2003.

NEPA - Núcleo de Estudos e Pesquisas em Alimentação. Tabela Brasileira de Composição de Alimentos.- T113. Versão II. 2. ed.Campinas: NEPA-UNICAMP, 2006. 113p.

SILVA, N.da.et al. Manual de Métodos de Análise Microbiológica de Alimentos. 3 ed. São Paulo: Varela, 2007.552p.

STONE, H.; SIDEL, J.L. Sensory evaluation pratices. 2 ed. San Diego: Academic Press Inc., 1993, 336p. 
